\title{
INDOOR LOCALIZATION FOR 3D MOBILE CADASTRAL MAPPING USING MACHINE LEARNING TECHNIQUES
}

\author{
C. Potsiou, N. Doulamis, N. Bakalos, M. Gkeli ${ }^{*}$, C. Ioannidis \\ Laboratory of Photogrammetry, School of Rural and Surveying Engineering, National Technical University of Athens, Greece; \\ chryssy.potsiou@gmail.com,ndoulam@cs.ntua.gr, bakalosnik@gmail.com, cioannid@survey.ntua.gr
}

KEY WORDS: 3D Cadastre, Crowdsourcing, 3D Mapping, Machine Learning, Indoor Localization

\begin{abstract}
:
With the rapid global urbanization, several multi-dimensional complex infrastructures have emerged, introducing new challenges in the management of the vertically stratified buildings spaces. 3D indoor cadastral spaces consist a zestful research topic as their complexity and geometry alterations during time, prevents the assignment of the corresponding Rights, Restrictions and Responsibilities (RRR). In the absence of the necessary horizontal spatial data infrastructure/floor plans their determination is weak. In this paper a fit-for-purpose technical framework and a crowdsourced methodology for the implementation of 3D cadastral surveys focused on indoor cadastral spaces, is proposed and presented. As indoor data capturing tool, an open-sourced cadastral mobile application for Android devices, is selected and presented. An Indoor Positioning System based on Bluetooth technology is established while an innovative machine learning architecture is developed, in order to explore its potentials to automatically provide the position of the mobile device within an indoor environment, aiming to add more intelligence to the proposed 3D crowdsourced cadastral framework. A practical experiment for testing the examined technical solution is conducted. The produced results are assessed to be quite promising.
\end{abstract}

\section{INTRODUCTION}

Over the last decades 3D Cadastre has been a major topic of interest, as it presents the spatial extent of ownership and determines the 3D property Rights, Restrictions and Responsibilities. Several 3D Cadastre attempts have been initiated world wide, as 3D information is essential for efficient land and property management (Gkeli et al., 2019a). Developments in computer graphics and 3D modelling techniques (McClunea et al., 2016; Köhn et al., 2016; Koeva and Oude Elberink, 2016; Gkeli and Ioannidis, 2018a) attracted the growing interest of researchers, making 3D cadastre technologically feasible. However, the implementation of a fully functional 3D Cadastre has not been achieved yet, due to the absence of integration between legal, institutional and technical parties involved (Koeva et al., 2019).

So far, most of the ongoing research on 3D Cadastre worldwide is focused on interrelations at the level of buildings. The analysis of cadastral indoor spaces, requires intensive research. The delimitation of 3D indoor cadastral objects is pretty challenging, as the geometry of the interior buildings spaces distinguished by complexity and may undergo a severe change over time. To record the geometry of $3 \mathrm{D}$ indoor cadastral spaces, a common practice is to use 2D plans for subdivisions and assignment of the rights, restrictions, and responsibilities to each one of the building spaces. However, the availability of 2D plans does not constitute the case for the majority of both new and old construction. With the advent of new technological achievements for indoor positioning, new potential opportunities arise, for the implementation of 3D Indoor Cadastre.

The immidiate development of a functional 3D cadastral system is of a great importance for both the developed and the developing world, as it ensures ownership rights, reduces risks, time and costs in urban property markets, and enables poverty reduction. This perception is contended by several actors such as UN-Habitat and the International Federation of Surveyors (FIG) (Lemmen et al., 2015). A fit-for-purpose 3D crowdsourced cadastral surveying approach seems to be the most appropriate solution, in order to meet the 2030 UN Agenda SDGs and ensure that both developed and developing countries may develop functional land administration systems fast and reliable with affordable costs, utilizing international standards (Enemark et al., 2014). Low-cost equipment, crowdsourcing techniques, machine learning techniques, automated procedures, mobile services (mservices), web services and open-source software (OSS) as well as standardized international data models, sign a new era for the cadastral data acquisition, recording, exchange and dissemination procedures, reducing the cost and the time of the required cadastral surveying (Cetl et al., 2019).

This paper presents a part of an on-going research project presented in Gkeli et al. (2019b), intended to provide a practical technical tool for the initial acquisition and management of 3D property rights mainly in urban areas. This work explores the opportunities for using innovative machine learning techniques and Bluetooth technology for Indoor Positioning in order to automatically acquire the position of indoor cadastral spaces, providing a plan-free solution, simplifying and adding more intelligence the $3 \mathrm{D}$ cadastral registration procedure. As a first attempt, a machine learning architecture similar with the one presented in Kaselimi et al. (2019b), is examined. The potentials and perspectives of this experiment, as well as our thoughts for future work, are discussed.

\section{RELATED WORK}

\subsection{Contemporary Practices for 3D Cadastres}

Since 2012, the main international framework for 3D Cadastres is the Land Administration Domaim Model (LADM ISO 19152, 2012). However, in LADM there is a lack of determinasion regarding the acceptable $3 \mathrm{D}$ geometries and representations for the $3 \mathrm{D}$ cadastral objects as well as the connection between widely known spatial models, such as Building Information Modeling (BIM) and IndoorGML (Rajabifard et al., 2019). Resolving these issues is pretty challenging, attracting the increasing attention of researchers (Ying et al., 2015). Several efforts have been 
performed in order to set a link between the legal and physical counterparts of 3D cadastral objects, utilizing several different technologies, application schemas and technical models, such as CityGML, IndoorGML, BIM/IFC, LandXML, InfraGML, etc. (Thompson et al., 2016; Atazadeh et al., 2018; Alattas et al., 2018; Gkeli et al., 2018b; Gkeli et al., 2019a). Furthermore, quite a few countries have tried to adopt the LADM standard, modifying and extending their local systems, aiming to develop a modern LADM-based 3D Cadastral Information System (Lee et al., 2015; Rajabifard et al., 2018; Gkeli et al., 2019a). However, these approaches are of high demands in terms of required time and financial resources.

In the meantime, the utilization of cutting-edge technologies and crowdsourcing techniques in developing $2 \mathrm{D}$ cadastral surveying procedures in order to minimize the necessary time and cost, has already been achieved (Basiouka and Potsiou, 2014; Mourafetis et al., 2015; Gkeli et al., 2016; Basiouka and Potsiou, 2016; Apostolopoulos et al., 2018; Potsiou et al., 2020; Mourafetis and Potsiou, 2020). A fit-for-purpose 3D crowdsourced cadastral surveying approach, based on the above advances, is of significant value in order to establish $3 \mathrm{D}$ cadastres, especially in the densely populated and self-developed cities. Research on this topic has focused on the investigation of applying the existing 2D experience in 3D cadastral surveys. Ellul et al. (2016) proposed the development of a web-based application that was intended to be used by the general public for the identification of the various land and property ownership situations. The user of the application is asked to select his/her situation from several groups including different types of land ownership, which where sketched by the research team. In Gkeli et al. (2017) a cost effective and fast procedure for 3D cadastral data acquisition and $3 \mathrm{D}$ visualization of the real properties, as block models (LoD1), on a mobile's phone screen at real-time, is presented. The developed prototype application aims to generate 3D building models through the digitization of property units' boundaries on the available basemap. As a basemap, a recent orthophoto of the area under cadastral survey, overlaid with 2D floor plans may be the best option for an Accurate, Assured and Authoritative (AAA) 3D cadastre (Williamson et al., 2012; Gulliver, 2015). Otherwise, other platforms, such as an aerial photo, may be used reducing the geometric accuracy significantly. In such case land parcel boundaries and building footprints should be visible. Gkeli et al. (2019a) present a similar approach that may be adopted both by the developed and developing countries, while the developed mobile application is able to manage and visualize 3D property models both above and below the land surface. The knowledge and the conclusions gained by this research are used to update the developed mobile application and establish a LADM-based database and an appropriate methodology, in order to proceed with $3 \mathrm{D}$ property unit registration and visualization exclusively through the developed application. The results seem to be promising, providing the basis for the implementation of a fit-forpurpose 3D cadastre. However, the proposed crowdsourced solutions (Gkeli et al., 2017; Gkeli et al., 2018b; Gkeli et al., 2019a; Gkeli et al., 2019b) rely on the basis of ideal conditions, assuming that the necessary horizontal spatial data infrastructure/floor plans are available. This may constitute the case for the majority of the relatively new constructions in the developed countries but is not widely applicable, as in many countries such plans may not be easily available. The existence of accurate basemaps both at ground level (land parcel boundaries) and at each building floor (indoor property unit boundaries at each floor) is necessary to achieve accurate geometric recording.

In the absence of accurate basemaps for the recognition and digitization of the land parcel boundaries, there are several options to proceed with their identification, such as (a) by using the smartphone's GPS sensor with an accuracy of a few meters, or (b) by using external support GNSS (Global Navigation Satellite System) tools and resources, achieving high positioning accuracy. Utilizing the Bluetooth capability of smart devices, different mobile GNSS receivers may be connected with smartphone's positioning applications, providing reliable and accurate results. For example, Esri's Collector Application may be connected with Trimble R2GPS GNSS receiver device, providing sub-meter accuracy for the observed points (Molendijk et al., 2018; Celt et al., 2019; Potsiou et al., 2020), or with EOS Arrow Gold RTK GNSS receiver device (EOS Arrow Gold RTK GNSS, 2020), providing even centimetre accuracy for the observed points. Furthermore, in the absence of accurate floor plans to be used as basemaps for digitization of the property unit boundaries in the indoor environment the 3D positioning problem at each floor is more challenging as the GPS/GNSS signal is weak and therefore indoor positioning relies typically on local infrastructure/measurements and other type of support.

\subsection{Machine Learning for Indoor Positioning Systems}

For indoor localization, several Indoor Positioning Systems (IPS) have been proposed, exploring different data sources. The most commonly utilized technologies use Bluetooth, ZigBee and Wi$\mathrm{Fi}$ as positioning signals. As it has been proven the hardware of such positioning technologies tends to be relatively cheap, making them preferable for low-cost location based applications (Zhang and Man, 2018).

The existing indoor position solutions use several different measurements and techniques to derive the desired location. The most common technique is fingerprinting, while there are several other techniques are also widely used, such as lateration, dead reckoning (Ibrahim et al., 2018). Fingerprinting is consisted by two separate phases: the offline and the online phase. In the offline phase, reference locations in the examined area are measured and stored in the database. In the online phase, the new measurements are compared with the ones stored in the database and then the system provides the real location of the receiver. Many of the existing fingerprint-based IPS exploit the Received Signal Strength (RSS) of the Wi-Fi, as it constitutes a simple solution with low hardware requirements. RSS fingerprints are usually combined with different machine learning methods to produce models, such as k-Nearest Neighbors (KNN), Neural Networks and Support Vector Machines (SVM) (Ibrahim et al., 2018).

The first fingerprinting localization system was presented in Bahl and Padmanabhan (2000) and was named RADAR. Laoudias et al. (2009) proposed a fingerprinting technique based on clustered Radial Basis Functions (RBF) Neural Network named (cRBF). The output representing the final values of the $\mathrm{x}$ and $\mathrm{y}$ coordinates. Zhang and Man (2018) proposed a Convolutional Neural Network (CNN) based architecture for Wi-Fi fingerprint positioning. The proposed $\mathrm{CNN}$-based architecture performs better on the public datasets than traditional machine learning methods. Furthermore, deep learning has been widely applied in various fields resulting in great performances. Zhang et al. (2016) proposed a 4-layer deep neural network (DNN) that generates coarse positioning estimate. The final position estimation is derived by a hidden Markov model (HMM) fine localizer. Wang et al. (2015) proposed a DBN (Deep Belief Networks) method, which applied the deep learning algorithm to fingerprint positioning for the first time. However, this implementation was weak, as the inappropriate parameter selection of DBN made the training process particularly difficult.

Combining these approaches and technologies, positional data may be processed in order to obtain the missing building's floor plans in a fit-for-purpose manner. The majority of current IPS 
solutions that automatically construct indoor floor plans with pervasively collected data, often rely on the inertial processing to infer the users' trajectories (Santos et al., 2019). Due to the manmade environmental noise these solutions are strongly affected, leading to several errors. For this reason, the utilization of different data sources is suggested. In Shin et al. (2012) a solution named SmartSLAM is proposed. SmartSLAM relies on the WiFi infrastructures available in most buildings by combining the signal patterns with the Simultaneous Localisation and Mapping (SLAM) techniques (Durrant-Whyte and Bailey, 2006). The indoor floor plans are gradually constructed through tracking the user's movement. Following a similar idea, Faragher and Harle (2013) proposed a smartSLAM scheme that contains Pedestrian Dead Reckoning (PDR), Fingerprint Extended Kalman Filter (FEKF), Fingerprint Extended Kalman Filter SLAM (FEKFSLAM) and Distributed Particle SLAM (DPSLAM).

\section{3D CADASTRAL INDOOR MAPPING}

This research tends to support and upgrade the technical solution for the compilation of 3D cadastral surveys, presented in Gkeli et al. (2019b), by enhancing the localization process in indoor environments. Our aim is to exploit the capabilities of wireless networks and mobile devices to provide an intelligent crowdsourced procedure model for the compilation of $3 \mathrm{D}$ indoor cadastral mapping.

\subsection{Background Information}

The main objective of the proposed crowdsourced approach is to provide a cost-effective, reliable and fast solution for the initial implementation of 3D cadastral systems. The role of citizens/right holders is essential, as they are not only asked to declare their rights but they simultaneously undertake the responsibility for the initial collection of the necessary geometric cadastral information. The technical framework supporting this venture consists of two connected sub-systems: (i) the LADMbased cadastral Database Management System (DBMS), where the collected data are stored and maintained, and (ii) the data capturing tool, that is a mobile device (e.g., smartphone) with a built-in cadastral application (Figure 1).

The application interface is appropriately configured in order to lead and simplify the registration procedure, facilitating its use by non-professionals (Figure 1). Also, provides a set of capturing tools for the identification and digitization of both land parcel's and building unit's boundaries on the available basemap. Simultaneously, allows the insertion of all necessary proprietary information, available documents (e.g., plans, deeds, etc.) and verification photos, for the declaration of the property rights. After inserting all the required geometric information concerning the building unit's/property's structure (height, floor, digitized boundaries) an automated algorithm following a model-driven approach, processes the declared geometric data and generates the 3D block models (LoD1) of both the land parcel and the property unit. The produced 3D property models may be visualized on the mobile's phone screen, both above or below the ground, by selecting each one of the visualization tools.

For the implementation of 3D cadastral surveys, a crowdsourced methodology is followed, consisted by five (5) main phases (Figure 2): (i) declaration of an area under cadastral survey by the government; collection of the available horizontal geospatial information for the preparation of a draft cadastral registration basemap; assignment of local team leaders to each sub-region by the municipality and the professional, (ii) citizens briefing, motivation and training by the team leaders; (iii) establishment of the available draft cadastral map of the area as basemap in the mobile application; in absence of professional floor plan, an orthophoto, an aerial photo or even an Open Street Map (OSM) may be used as a registration basemap; technical and IT preparation by the team leaders for each multi-story building in collaboration with the manager of each building, (iv) 3D cadastral data acquisition by the rights holders/citizens, and (v) data evaluation, control and submission of the missing data by the rights holders/citizens; compilation of preliminary $3 \mathrm{D}$ cadastral database.

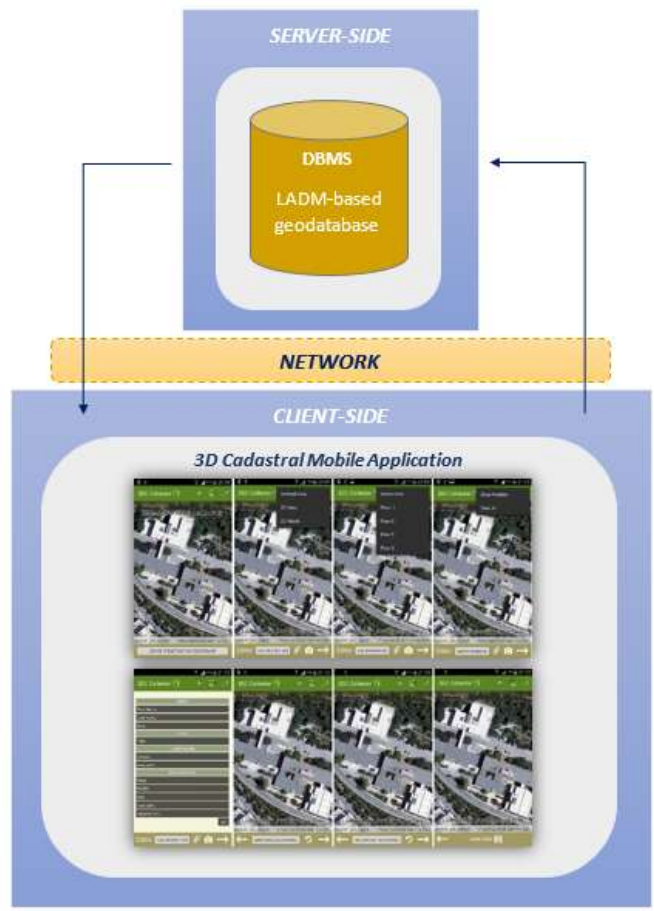

Figure 1. Architecture diagram of the 3D cadastre technical framework

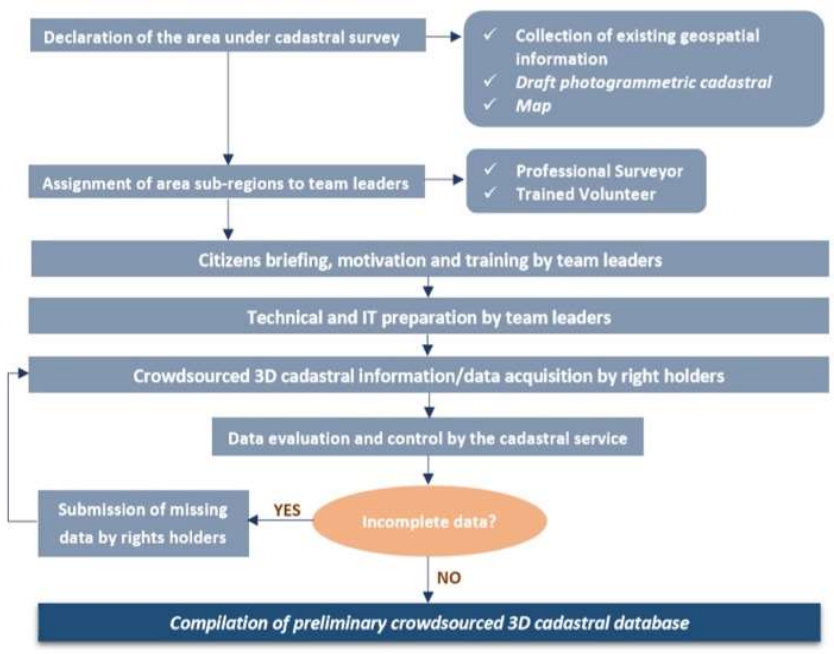

Figure 2. Crowdsourcing methodology for 3D cadastral surveys (Gkeli et al., 2019b)

\subsection{Proposed Cadastral System Upgrade}

The proposed upgrade aim to reform, simplify and strengthen one of the most demanding phases of the implementation process, which strongly affects the quality and reliability of the final product. This is the fourth phase of the proposed methodology, concerning the acquisition of the necessary data by the right holders. As the majority of right holders are non-professionals, 
their orientation in 3D space and therefore the correct digitization of their property boundaries on the basemap, consist a complex procedure, but of great importance for the proper and reliable cadastral registration.

An Indoor Positioning System (IPS) based on low-cost Bluetooth technology in combination with the 3D cadastral mapping mobile application are the two main pillars supporting the proposed modification and upgrade of this framework. The main idea of the designed IPS solution is to gradually construct the plan of indoor cadastral spaces/property boundaries, through tracking the mobile device's/user's trajectory. Through the cadastral mobile application and the smartphone's Bluetooth wireless sensor, right holders may 'visit' their property and delineate the boundaries of their indoor cadastral spaces on the basemap, utilizing the established IPS. By processing the received IPS signals through a machine learning algorithm the generation of horizontal spatial information, regarding the position of the mobile device in the indoor environment may be provided and visualized through the cadastral mobile application (Figure 3). Thus, right holders may gradually construct the boundaries of their property unit (indoor cadastral space), by moving towards the physical boundaries of their property and digitize them by selecting their corresponding position on the basemap.

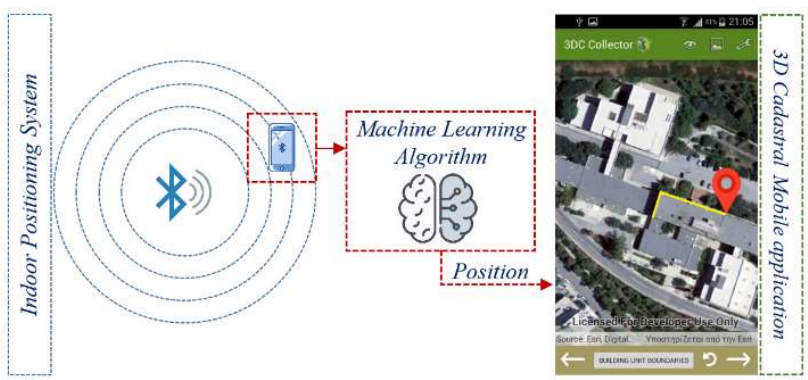

Figure 3. 3D cadastral indoor mapping - Framework overview

\section{PROPOSED MACHINE LEARNING ARCHITECTURE}

The purpose of the machine learning architecture is to estimate the coordinates of a space using information provided by bluetooth sensors. In the following, the bluetooth sensing interface and the proposed deep learning framework for processing and analysing the signals from the bluetooth sensors in order to estimate space coordinates are briefly described.

\subsection{Sensing interface}

The main sensing modality for the indoor localization are multiple Bluetooth signals. Bluetooth is a wireless technology, standardised as IEEE 802.15.1, that uses short-wavelength UHF radio waves. It operates between 2.402 and $2.480 \mathrm{GHz}$, including $2 \mathrm{MHz}$ wide guard bands. Bluetooth by itself has no location intelligence built into it; they rather act as lighthouse beacons transmitting signals in the area of coverage. The capturing device is responsible for capturing the signal and understand its meaning. However, by assigning these beacons with physical coordinates, you can compare the received signal. The spatial correlation can be achieved using the Received Signal Strength Indicator (RSSI). RSSI is an indication of the power level of the signal received from an antenna. An overview of the end-device beacon protocol profile can be viewed in Figure 4 .

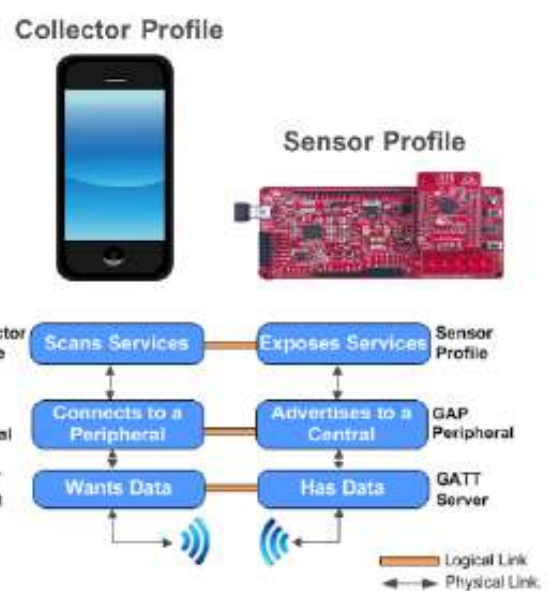

Figure 4. Overview of the Beacon/Collector profile

RSSI signal can only give a rough estimation of the distance between the beacon and end-device, as Bluetooth does not have a directional antenna. However, by multiple RSSI values from beacons the localisation error can be minimised and cm-level accuracy of position can be achieved. In this work, we utilise the publicly available dataset of Baronti et al. (2018), that captures Bluetooth signals from 8 different Bluetooth beacon to localize the signal for 8 different rooms and a small corridor. A more detailed description can be found in section 5 .

\subsection{The deep learning framework for coordinate estimation}

The purpose of this module is to estimate the coordinates of the space using distortions of the signal of the bluetooth sensors. In particular, let us denote as $b_{i}(t)$ the bluetooth signal of the $i$-th sensor. In case that we have Bluetooth available sensors, we have also $N$ available distortion signals $b_{i}(t), i=1,2, \ldots N$. Distortions of signals $b_{i}(t)$ provides an estimation of the space coordinates. In particular, let us denote as $\mathbf{s}_{c}$ a vector contains the space coordinates of certain space positions. Therefore, we have that $\mathbf{s}_{c}=\left[s_{c}\left(p_{1}\right), s_{c}\left(p_{2}\right), \ldots, s_{c}\left(p_{K}\right)\right]^{T}$. In this notation, $K$ denotes the number of space coordinates, the algorithm estimates and $s_{c}\left(p_{i}\right)$, the respective coordinate at position $p_{i}$ out of the $K$ available positions. It is clear that the captured distortion signals $b_{i}(t)$ is related with the space position estimates $\mathbf{s}_{c}$, using a non-linear relationship of

$$
\mathbf{s}_{\mathbf{c}}=f\left(b_{1}(t), \ldots, b_{N}(t)\right)
$$

In Eq. (1) function $f(\cdot)$ expresses a non-linear relationship that correlates the distortion signals $b_{i}(t)$ with the space coordinate vector $\mathbf{s}_{c}$.

The main difficulty of modelling Eq. (1) is that the non-linear function $f(\cdot)$ is actually unknown. One way to model the unknown function $f(\cdot)$ of Eq. (1) is by means of a Feedforward Neural Network (Doulamis et al., 2003). Assuming $L$ hidden neurons, each of response $u_{j, i}$ - referring to the $s_{c}\left(p_{j}\right)$ space location of the vector $\mathbf{s}_{c}$ - and one linear output layer, the estimate $s_{c}\left(p_{j}\right)$ is given by

$$
\begin{gathered}
s_{c}\left(p_{i}\right)=\mathbf{u}_{j}(t)^{T} \cdot \mathbf{v}_{j} \\
\mathbf{u}_{j}(t)=\left[\begin{array}{c}
u_{j, 1}(t) \\
\vdots \\
u_{j, L}(t)
\end{array}\right]=\left[\begin{array}{c}
\tanh \left(\mathbf{w}_{j, 1}^{T} \cdot \mathbf{b}(t)\right) \\
\vdots \\
\tanh \left(\mathbf{w}_{j, L}^{T} \cdot \mathbf{b}(t)\right)
\end{array}\right]
\end{gathered}
$$

$\tanh (\cdot)$ refers to the hyperbolic tangent, so each element of the 
vector $\mathbf{u}_{j}(n)$ ranges between -1 and 1 . The weights $\mathbf{w}_{j, i}, i=1, \ldots, L$, connect the input vector $\mathbf{b}(t)=\left[b_{1}(t) \cdots b_{N}(t)\right]^{T}$ with the $i$-th hidden neuron. Similarly, $\mathbf{v}_{j}$ are the weights that connect the hidden neurons with the output neuron. Vector $\mathbf{u}(\mathrm{n})$ gathers the outputs of all $L$ hidden neurons $u_{j, i}$ and is a state vector expressing latent operational modes of the $j$-th space position estimate. Their values range within the $[-1,1]$ interval. Each space position estimate $s_{c}\left(p_{j}\right)$ has its own hidden states and these latent variables are estimated through a learning process. Since we assume that all the processing refers to the $j$-th space location, that is of estimate the scalar value $s_{c}\left(p_{j}\right)$ of vector $\mathbf{s}_{c}$, in the following analysis subscript $j$ is omitted for simplicity.

Since the space positioning $s_{c}\left(p_{j}\right)$ follows a non-causal relationship, the value of a state $u_{i}(t)$ depends not only on its previous values and but also on its future ones (Figure 5). Therefore, we have that

$$
u_{i}(n t)=\tanh \left(\mathbf{w}_{i}^{\mathrm{T}} \cdot \mathbf{b}(t)+\overrightarrow{\mathbf{r}}_{i}^{\mathrm{T}} \cdot \mathbf{u}(t-1)+\cdot \overrightarrow{\mathbf{r}}_{i}^{\mathrm{T}} \cdot \mathbf{u}(t+1)\right)
$$

In Eq. (3), $\overrightarrow{\mathbf{r}}_{i}$ is a weight vector expressing the dependencies of previous states, while $\hat{\mathbf{r}}_{i}$ the future state dependencies (Graves, 2013).

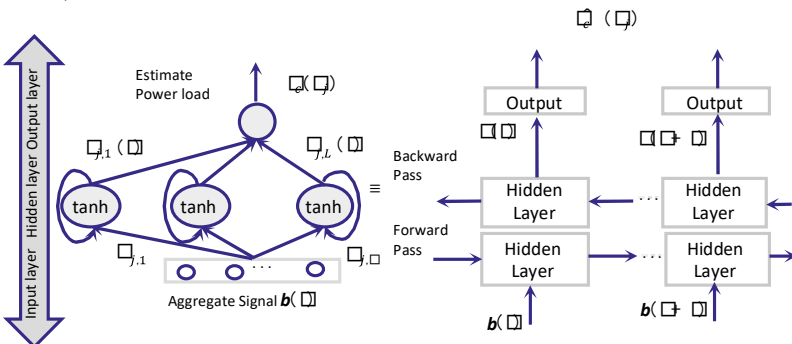

Figure 5. The architecture of a recurrent neural network model with bi-directional capabities (Kaselimi et al., 2019b)

\subsection{Long-range bi-directional dependence}

Usually a short-range dependent, that is of one forward or backward pass, is not adequate for modelling the unknown function $f(\cdot)$. For this reason, a long-range dependent modelling framework is adopted, having also bi-directional (i.e., noncausal) capabilities. Therefore, a bi-directional Long-Short Term Memory (LSTM) network is adopted as the basic regression model for power load estimation (Kaselimi et al., 2019a). These models are of similar structure to the bi-directional recurrent regression models but each node in the hidden layer is replaced by a memory cell, instead of a single neuron. The architecture of a bi-directional LSTM network is depicted in Figure 6.

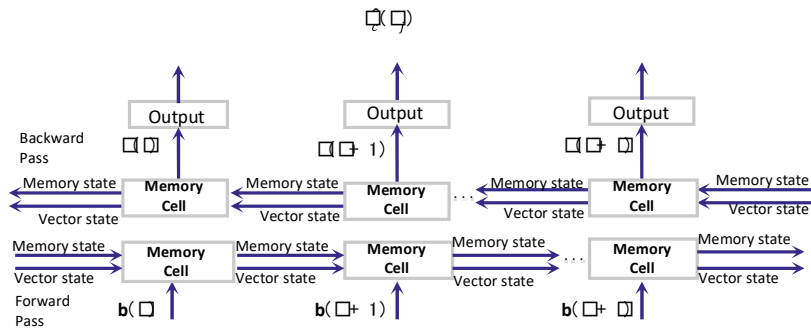

Figure 6. The architecture of a bi-directional neural network model used for estimating the space coordinates for the distrortion of the bluetooth signals $b_{i}(t)$
The memory cell contains three different components: i) the forget gate, ii) the input gate and the input node, and iii) the output gate. The architecture of the memory cell is presented in Figure 7.

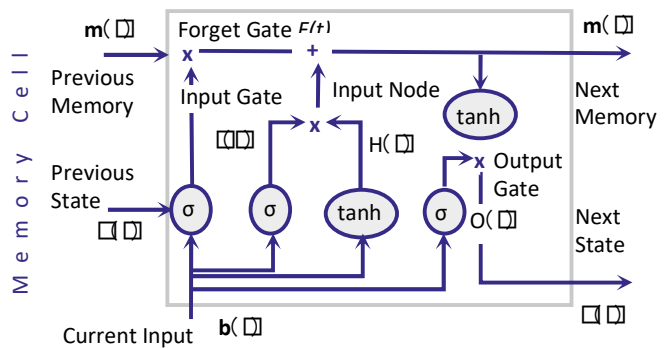

Figure 7. The architecture of the memory cell used in a bidirectional LSTM model (Kaselimi et al., 2019b)

The forget gate: The purpose of this component is to decide what information is throw out of the memory cell. The output ranges between 0 and 1 , due to the sigmoid activation function (function " $\sigma$ " in Figure 7). Values close to 0 means to dispose the incoming information [composed by the current power $\mathbf{b}(t)$ and the state vector $\mathbf{u}(t-1) / \mathbf{u}(t+1)$ ], while values close to 1 indicates that this information should be taken into consideration by the current memory cell.

The input node / Gate: The input node performs the same operation as the hidden neuron of the short-range model; it appropriately activates the respective state (true or false output from the "tanH" activation). Instead, the input gate regulates whether the respective hidden state is "significant enough" on the regression model; sigmoid operation.

The output gate: This regulates whether the response of the current memory cell is "significant enough" to contribute to the next memory cell.

Therefore, the operation of all the aforementioned modules is mathematically formulated by

$$
\begin{gathered}
\{F(n), H(n), I(n), O(n)\}=\{\sigma, \tanh \}( \\
\left.\mathbf{w}^{T,\{F, H, I, O\}} \cdot \mathbf{b}(t)+\overrightarrow{\mathbf{r}}^{T,\{F, H, I, O\}} \cdot \mathbf{u}(t-1)+\dot{\mathbf{r}}_{j, i}^{T,\{F, H, I, O\}} \cdot \mathbf{u}_{j}(t+1)\right)
\end{gathered}
$$

\subsection{Bayesian Optimization}

A Bayesian optimization strategy is adopted for selecting the best parameters of the bi-directional LSTM strategy. The proposed methodology is similar to the approach discussed in Kaselimi et al. (2020). In this way, the machine learning regression approach in estimating the space coordinates from the distortions of the bluetooth signals is optimized. Therefore, the precision accuracy, as far as space positioning is concerned, is increased. The scope of Bayesian optimization is to estimate a better configuration parameter regarding network classification from the current parameter selection. This mean that the algorithm selects a configuration that minimizes the regression error $E$ of the machine learning network. Since, we do not know exactly the mathematical form of the error $E$, a Baysesian strategy is adopted. The strategy assumes that the regression error $E$ follows a Gaussian distribution. Therefore, selection of the best parameter values is the ones that minimizes the overall error $E$. 


\section{APPLICATION}

The proposed machine learning framework is benchmarked over the publicly available dataset of Baronti et al. (2018). The test environment is consisted from seven contiguous rooms and a connecting corridor as well as a small adjacent area. The overview of the area is depicted in Figure 8. The total area covered amounts to $185 \mathrm{~m}^{2}$ with a maximum horizontal span of $16.6 \mathrm{~m}$ and a maximum vertical span of ca. $14.3 \mathrm{~m}$.

The methodology for placing the Bluetooth beacons that was followed, included one Bluetooth beacon per room, placed in the middle of the wall on the fire side of the room's entrance. An overview of the beacons and number of locations captured can be viewed in Figure 8 (the beacons have a 4digit ID and are colored yellow in the figure).

Specifically, the survey scenario of the dataset was adapted to our proposed Bidirectional LSTM classifier. In our experimental setup, each target position label has a granularity accuracy of $60 \mathrm{~cm}$, in order to simplify the data capturing and measurement effort.

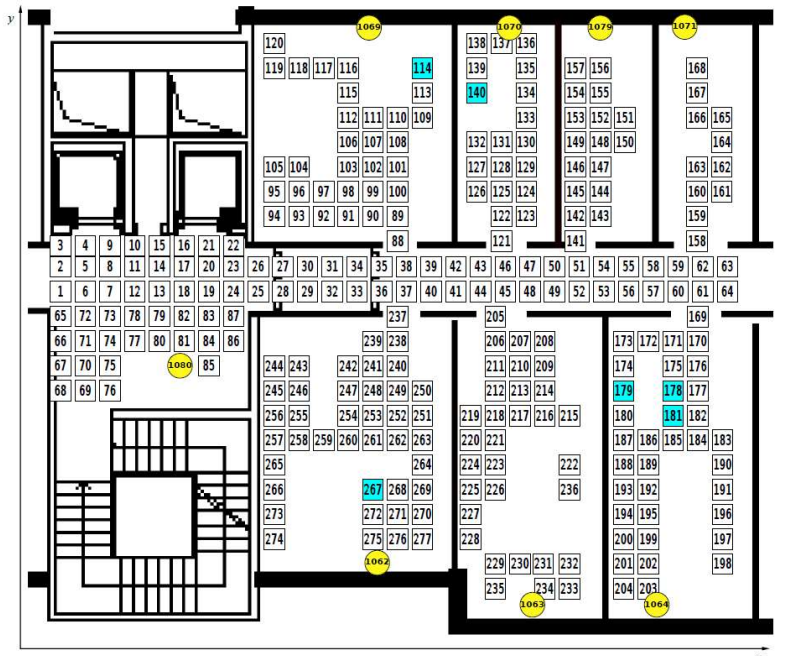

Figure 8. Overview of the test environment

The proposed LSTM classifier was implemented in Python 3.6 using the Keras (1.08) and Tensorflow (2.1.0) machine learning libraries, in combination with a number of other scientific and data management libraries. The model was trained using a Intel Core i7-6700K CPU (4GHz) with 2 NVIDIA GTX1080 GPUs. The tested LSTM classifier consisted, beyond the necessary input and output layers, with 2 LSTM Layers with rectified linear unit (ReLU) activations and 0.2 dropout between each layer.

Performance evaluation has been performed among the Bayes Bidirectional LSTM network and two other approaches: a CNN and a unidirectional LSTM network. The time required for training the network is of about $8 \mathrm{hr}$, with the PC setup described above. However, the computation time for estimating a position, after the model is trained, is less than $1 \mathrm{sec}$, which is appropriate for such an application scenario. The performance of the tested frameworks can be found in Table 1. For the performance evaluation the typical machine learning metrics, that is, accuracy, precision, recall and F1-score are used. The mean squared errors of the framework can be viewed in Figure 9.

As observed, the LSTM frameworks outperform the CNN one. Between the two LSTM approached the bidirectional one attains the minimum error since it is capable to model non-causal behaviour. The average accuracy estimating a target position label is about $12 \mathrm{~cm}$. This is significantly less than the average accuracy of the CNN, which was approximately $22 \mathrm{~cm}$, and the unidirectional LSTM, that had a precision of approximately
$16 \mathrm{~cm}$. Therefore, the proposed architecture reduces the granularity accuracy by a factor of about 5 . More precise target labels will further increase this frameworks accuracy.

\begin{tabular}{l|c|c|c} 
& CNN & LSTM & BiLSTM \\
\hline Accuracy & $72.16 \%$ & $79.87 \%$ & $84.14 \%$ \\
\hline Precision & $58.04 \%$ & $71.67 \%$ & $78.04 \%$ \\
\hline Recall & $95.78 \%$ & $88.03 \%$ & $88.26 \%$ \\
\hline F1 & $72.28 \%$ & $79.01 \%$ & $82.84 \%$ \\
\hline
\end{tabular}

Table 1: Performance Metrics of the tested classifiers

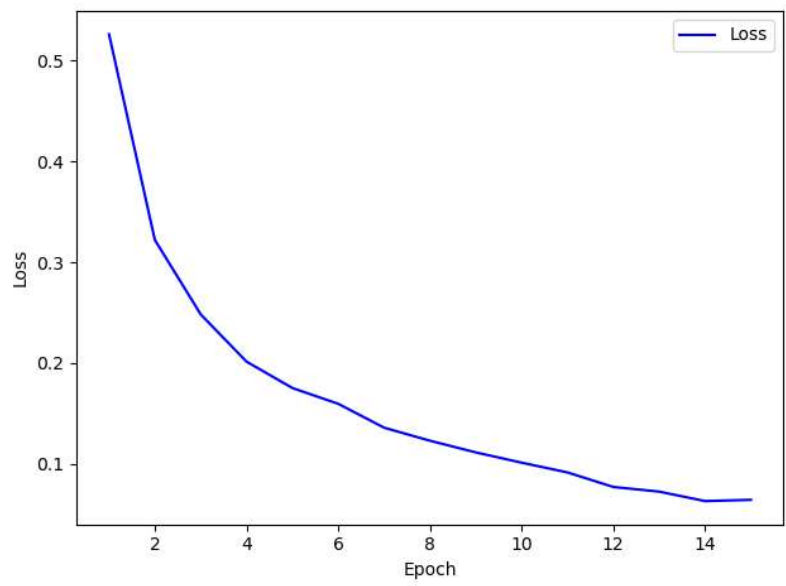

Figure 9. Per epoch loss for the optimized bidirectional LSTM

The overall method provides a number of advantages, as it achieves cm-level accuracy using a sensing framework that is easily deployed. This is due to the fact that Bluetooth beacons are relatively cheap and mobile (an off the shelf device has dimensions of only a few centimeters), and can be easily installed in the location.

\section{DISCUSSION AND CONCLUSIONS}

This work is part of an on-going project aiming to develop a technical tool and a methodology for the future collection, management and 3D modelling of cadastral data. The introduction of crowdsourcing and mobile devices as data capturing tools facilitate the registration procedure, allowing the right holders to move throughout the property, using the mobile device's integrated sensors, and collect the necessary information/measurements. Thus, the duration of field cadastral surveys by professionals, is reduced significantly. So far, the performance of the presented mobile application and methodology for 3D cadastral data registration, using an accurate/professional basemap (such as architectural floor plans), has led to satisfying results, both reliable and affordable. However, in the absence of such an accurate basemap the identification and digitization of the property unit boundaries in the indoor environment is challenging, as the GPS/GNSS signal is weak, introducing large positioning errors.

The confrontation of indoor positioning weakness, in the absence of an accurate basemap is of great importance. The research towards investigating new methods for easy and reliable property unit boundary coordinate measurement by the right holders is at an initial stage and it presents a proposal for the potential integration of innovative machine learning techniques and Bluetooth technology for Indoor Positioning, in the proposed 
technical system for the implementation of $3 \mathrm{D}$ cadastral surveys. In this paper, using Bluetooth signal strength and a bi-directional LSTM network, with optimised hyperparameters, an accurate indoor localisation service is structured and presented. The performance of the proposed deep learning framework is compared with other popular frameworks, namely a CNN and a unidirectional LSTM network. The proposed Bayesian optimised bi-directional LSTM outperforms the other classifiers, providing reliably accurate space positioning which must be further exploited by the presented mobile application, thus expanding the potentials of indoor 3D cadastral mapping. For future work, more complex deep machine learning architectures such as multichannel recurrent neural networks (Kaselimi et al., 2019b) or deep NARMA filters (Bakalos, 2019) should be investigated. These structures, are capable of (i) processing simultaneously signals from heterogeneous sensors (e.g., Bluetooth and $\mathrm{WiFi}$ signals) in order to increase indoor localisation accuracy (multichannel RNNs) and (ii) of introducing an autoregressive behaviour to the LSTM neural network structure for further improvements in precision accuracy. In addition, as future work we can reduce the granularity of the targets positions labels in order to further improve the proposed architecture. Another extension is to incorporate in the sensing infrastructure, apart from Bluetooth sensors, Channel State Information of WiFi signals, which has been studied as an additional localisation modality (Bakalos, 2019).

The next step of this research will be the integration between the developed indoor positioning system and the cadastral mobile application. A practical application of the optimized system will be implemented, in order to investigate potentials and weaknesses of the proposed solution, providing some key conclusions regarding the achieved geometric accuracy, system's usability, registration duration and cost of the proposed system's infrastructure. However, first results derived from the proposed system have shown promising potential for the development of a "plan-free" solution, simplifying the 3D cadastral registration procedure and speeding up the processes for the immidiate implementation of a fit-for-purpose 3D cadastral system. Another future step for this application will be the training of a model from multiple buildings in order to increase accuracy, as well as determining the minimum number of bluetooth beacons necessary for reaching the performance required. Finally, generative approaches should be studied, in order to create a framework that can be used even in furnished places, where the user does not have physical access to the borders of the room. Such approaches could be used to generate the "unfurnished" model from a smaller model where there are obstacles in some areas of the room.

\section{ACKNOWLEDGEMENTS}

The contribution of Maria Gkeli to this research is part of her $\mathrm{PhD}$ dissertation, which is supported by the Onassis Foundation scholarship program. The authors would like to thank Ordnance Survey GB (https://www.ordnancesurvey.co.uk) and 1Spatial (https://1spatial.com/) for sponsoring the publication of this paper.

\section{REFERENCES}

Alattas, A., van Oosterom, P., Zlatanova, S., 2018. Deriving the Technical Model for the Indoor Navigation Prototype based on the Integration of IndoorGML and LADM Conceptual Model. 7th International FIG Workshop on the Land Administration Domain Model, 245-268.
Apostolopoulos, K., Geli, M., Petrelli, P., Potsiou, C., Ioannidis, C., 2018. A new model for Cadastral Surveying using Crowdsourcing. Survey Review, 50(359), 122-133.

Atazadeh, B., Rajabifard, A., Kalantari, M., 2018. Connecting LADM and IFC Standards - Pathways towards an Integrated Legal-Physical Model. 7th International FIG Workshop on the Land Administration Domain Model, 89-102, Zagreb, Croatia.

Bahl, P., Padmanabhan, V.N., 2000. Radar: An in-building rfbased user location and tracking system. INFOCOM 2000. Nineteenth Annual Joint Conference of the IEEE Computer and Communications Societies, 2, 775-784.

Bakalos, N., Voulodimos, A., Doulamis, N., Doulamis, A., Ostfeld, A., Salomons, E., Caubet, J., Jiménez, V., Li, P., 2019. Protecting water infrastructure from cyber and physical threats: Using multimodal data fusion and adaptive deep learning to monitor critical systems. IEEE Signal Processing Magazine, 36(2), 36-48.

Baronti, P., Barsocchi, P., Chessa, S., Mavilia, F., Palumbo, F., 2018. Indoor Bluetooth low energy dataset for localization, tracking, occupancy, and social interaction. Sensors, 18(12), 4462.

Basiouka, S., Potsiou, C., 2014. The volunteered geographic information in cadastre: Perspectives and citizens' motivations over potential participation in mapping. GeoJournal, 79(3), 343355.

Basiouka, S., Potsiou, C., 2016. A Proposed Crowdsourcing Cadastral Model: Taking Advantage of Previous Experience and Innovative Techniques. European Handbook of Crowdsourced Geographic Information, Eds: Capineri, C., Haklay, M., Huang, H., Antoniou, V., Kettunen, J., Ostermann, F., Purves, R. Ubiquity Press: London, UK, 419-433.

Cetl, V., Ioannidis, C., Daylot, S., Doytsher, Y., Felus, Y., Haklay, M., Mueller, H., Potsiou, C., Rispoli, E., Siriba, D., 2019. New Trends in Geospatial Information: The Land Surveyors Role in the Era of Crowdsourcing and VGI - Current state and practices within the land surveying, mapping and geo-science communities. FIG Publication No 73, Copenhagen, Denmark.

Doulamis, A, Doulamis, N, Kollias, S, 2003. An adaptable neural-network model for recursive nonlinear traffic prediction and modeling of MPEG video sources. IEEE Trans on Neural Networks, 14(1), 150-166.

Durrant-Whyte, H., Bailey, T., 2006. Simultaneous localization and mapping: Part I. IEEE Robot. Autom. Mag. 2006, 13, 99110.

Ellul, C., de Almeida, J.P., Romano, R., 2016. Does coimbra need a $3 \mathrm{~d}$ cadastre? Prototyping a crowdsourcing app as a first step to finding out. ISPRS Ann. Photogramm. Remote Sens. Spatial Inf. Sci., IV-2/W1:55-62, https://doi.org/10.5194/isprsannals-IV-2-W1-55-2016

Enemark, S., Bell, K.C., Lemmen, C., McLaren, R., 2014. Fitfor-Purpose Land Administration. Joint FIG / World Bank publication, FIG Publication No 60, Copenhagen, Denmark. EOS Arrow Gold RTK GNSS, 2020. EOS Arrow Gold RTK GNSS receiver with SafeRTK. https://eosgnss.com/product/arrow-series/arrow-gold (March 2020).

Faragher, R., Harle, R., 2013. SmartSLAM - An efficient smartphone indoor positioning system exploiting machine learning and opportunistic sensing. 26th Int. Technical Meeting of the Satellite Division of the Institute of Navigation, Nashville, TN, USA.

Gkeli, M., Apostolopoulos, K., Mourafetis, G., Ioannidis, C., Potsiou, C., 2016. Crowdsourcing and mobile services for a fitfor-purpose Cadastre in Greece. Fourth International Conference on Remote Sensing and Geoinformation of the Environment (RSCy2016), SPIE, 9688, 17 p., doi:10.1117/12.2240835.

Gkeli, M., Ioannidis, C., Potsiou, C., 2017. VGI in 3D Cadastre: A Modern Approach. FIG Commission 3 - Annual Workshop 
"Volunteered Geographic Information: Emerging Applications in Public Science”, Lisbon, Portugal, $21 \mathrm{p}$.

Gkeli, M., Ioannidis, C., 2018a. Automatic 3d reconstruction of buildings roof tops in densely urbanized areas. Int. Arch. Photogramm. Remote Sens. Spatial Inf. Sci., XLII-4/W10, 47-54. https://doi.org/10.5194/isprs-archives-XLII-4-W10-47-2018.

Gkeli, M., Potsiou, C., Ioannidis, C., 2018b. LADM-based Crowdsourced 3D Cadastral Surveying - Potential and Perspectives. 6th International FIG Workshop on 3D Cadastres, Delft, Netherlands.

Gkeli, M., Potsiou, C., Ioannidis, C., 2019a. Crowdsourced 3D cadastral surveys: looking towards the next 10 years. $J$ Geogr Syst, 21, 61-87. https://doi.org/10.1007/s10109-018-0287-0

Gkeli, M., Potsiou, C., Ioannidis, C., 2019b. A technical solution for 3D crowdsourced cadastral surveys. Land Use Policy (in press). https://doi.org/10.1016/j.landusepol.2019.104419

Graves, A., 2013. Generating Sequences with Recurrent Neural Networks. arXiv:1308.0850 [cs].

Gulliver, T., 2015. Developing a 3D Digital Cadastral System for New Zealand. Master's Thesis, Department of Geography, University of Canterbury, Christchurch, New Zealand.

Ibrahim, M., Torki, M., El Nainay, M., 2018. CNN based Indoor Localization using RSS Time-Series. IEEE Symposium on Computers and Communications (ISCC), 1044-1049.

Kaselimi, M., Doulamis, N., Doulamis, A., Voulodimos, A., Protopapadakis, E. 2019a. Bayesian-optimized bidirectional LSTM regression model for non-intrusive load monitoring. IEEE Conference on Acoustic Speech and Singal Processing (ICASSP), 2747-2751.

Kaselimi, M., Protopapadakis, E., Doulamis, N., Doulamis, A., Voulodimos, A., 2019b. Multi-Channel Recurrent Convolutional Neural Networks for Energy Disaggregation. IEEE Access, 7, 81047-81056.

Kaselimi, M., Doulamis, N., Voulodimos, A., Protopapadakis, E., Doulamis, A., 2020. Context Aware Energy Disaggregation using Adaptive Bidirectional LSTM Models. IEEE Transactions on Smart Grid.

Koeva, M., Oude Elberink, S., 2016. Challenges for Updating 3D Cadastral Objects using LiDAR and Image-based Point Clouds. 5th International FIG Workshop on 3D Cadastre, Athens, Greece, 169-182.

Koeva, M., Nikoohemat, S., Oude Elberink, S., Morales, J., Lemmen, C., Zevenberger, J., 2019. Towards 3D Indoor Cadastre Based on Change Detection from Point Clouds. Remote Sensing, 11(17), 1972.

Köhn, A., Tian, J., Kurz, F., 2016. Automatic Building Extraction and Roof Reconstruction in 3k Imagery Based on Line Segments. Int. Arch. Photogramm. Remote Sens. Spatial Inf. Sci., XLI-B3, 625-631.

LADM ISO 19152, 2012. Land Administration Domain Model. http://www.iso.org/iso/home/store/catalogue tc/catalogue detai 1.htm?csnumber=51206 (December 2019).

Laoudias, C., Kemppi, P., Panayiotou, C.G., 2009. Localization using radial basis function networks and signal strength fingerprints in wlan. Global Telecommunications Conference 2009 (GLOBECOM 2009), IEEE, 1-6.

Lee, B.M., Kim, T.J., Kwak, B.Y., Lee, Y.H., Choi, J., 2015. Improvement of the Korean LADM Country Profile to build a 3D Cadastre Model. Land Use Policy, 49, 660-667.

Lemmen, C., van Oosterom, P., Bennett, R., 2015. The land administration domain model. Land Use Policy, 49, 535-545.

McClunea, A.P., Millsa, J.P., Millerb, P.E., Hollandc, D.A., 2016. Automatic 3d Building Reconstruction from a Dense Image Matching Dataset. Int. Arch. Photogramm. Remote Sens. Spatial Inf. Sci., XLI-B3, 641-648.

Molendijk, M., Dukon, T., Lemmen, C., Morales, J., Endo, V., Rodriguez, S., Dueñas, J., Sanchez, I., Spijkers, P., Unger, E.,
Horta, I., 2018. Land and Peace in Colombia: FFP Methodology for Field Data Collection and Data Handling. World Bank Land and Poverty Conference 2018, Washington DC, USA.

Mourafetis, G., Apostolopoulos, K., Potsiou, C., Ioannidis, C., 2015. Enhancing Cadastral Survey by Facilitating Owners' Participation. Survey Review, 47(344), 316-324.

Mourafetis, G., Potsiou, C., 2020. IT Services and Crowdsourcing in Support of the Hellenic Cadastre: Advanced Citizen Participation and Crowdsourcing in the Official Property Registration Process. ISPRS Int. J. Geo-Inf. 2020, 9(4), 190. https://doi.org/10.3390/ijgi9040190

Oldfield, J., van Oosterom, P., Quak, W., Veen, J., Beetz, J., 2016. Can Data from BIMs be Used as Input for a 3D Cadastre? 5th International FIG 3D Cadastre Workshop, Athens, Greece, 199-214.

Potsiou, C., Paunescu, C., Ioannidis, C., Apostolopoulos, K., Nache, F., 2020. Reliable 2D Crowdsourced Cadastral Surveys: Case Studies from Greece and Romania. ISPRS Int. J. Geo-Inf., 9(2), 89, $23 \mathrm{p}$.

Rajabifard, A., Agunbiade, M., Kalantari, M.M., Yip, KM., Atazadeh, B., Badiee, F., Isa, D., Adimin, M.K., Chan, K.L., Aien, A., Olfat, H., Shojaei, D., Anaraki, M.R., 2018. An LADMbased Approach for Developing and Implementing a National 3D Cadastre - A Case Study of Malaysia. Land Administration Domain Model Workshop, 47 - 66.

Rajabifard, A., Atazadeh, B., Kalantari, M., 2019. BIM and urban land administration. Taylor \& Francis, CRC Press.

Santos, R., Barandas, M., Leonardo, R., 2019. Fingerprints and floor plans construction for indoor localisation based on crowdsourcing. Sensors, 19(4), 919.

Shin, H., Chon, Y., Cha, H., 2012. Unsupervised Construction of an Indoor Floor Plan Using a Smartphone. IEEE Trans. Syst. Man Cybern. Part C Appl. Rev. 2012, 42, 889-898.

Thompson, R., van Oosterom, P., Soon, K., Priebbenow, R., 2016. A Conceptual Model Supporting a Range of 3D Parcel Representations through all Stages: Data Capture, Transfer and Storage. FIG Working Week 2016, 23 p.,

https://www.fig.net/resources/proceedings/2016/2016_3dcadastr e/3Dcad 2016_02.pdf.pdf

Wang, X., Gao, L., Mao, S., Pandey, S., 2015. DeepFi: Deep learning for indoor fingerprinting using channel state information. 2015 IEEE Wireless Communications and Networking Conference (WCNC) IEEE, 1666-1671.

Williamson, I., Rajabifard, A., Kalantari, M., Wallace, J., 2012. AAA Land Information: Accurate, Assured and Authoritative. 8th FIG Regional Conference 2012 Surveying towards Sustainable Development, Montevideo, Uruguay, 26-29.

Ying, S., Guo, R., Li, L., van Oosterom, P., Stoter, J., 2015. Construction of 3D Volumetric Objects for a 3D Cadastral System. Transactions in GIS, 19(5), 758-779.

Zhang, W., Liu, K., Zhang, W., Zhang, Y., Gu, J., 2016. Deep neural networks for wireless localization in indoor and outdoor environments. Neurocomputing, 194, 279-287.

Zhang, T., Man, Y., 2018. The enhancement of WiFi fingerprint positioning using convolutional neural network. Int. Conf. Comput., Commun. Netw. Technol. (CCNT), Wuzhen, China. 